\title{
The dangers of wearing dental gloves near open flames - a study of five brands
}

\author{
An evaluation of the flammability of five dental gloves by R. G. Chadwick, F. M. Tulley Br Dent J2001; 190:561-562
}

\section{Objective}

To assess the flammability of five brands of dental procedure glove.

\section{Design \\ A total of ten gloves of each brand (Biogel-D, Premier Protectors, Roeko-D, Safeskin Satin Plus and Schottlander Low Allergy) underwent flammability testing. Five of these were tested as supplied (unwashed) and five following the application of the hand disinfectant Hydrex (washed). Each glove was stretched over a metal frame and the time to ignition, when exposed to a standardised butane flame, recorded. In addition, the thickness of each glove was also assessed by micrometer measurement.}

\section{Results}

All the gloves tested ignited in less than 2.5 seconds. Two way analysis of variance revealed significant effects of glove type $(P<$ $0.001)$ and treatment $(P<0.05)$, together with a significant interaction of these factors $(P<0.05)$, upon the ignition time. Washing Roeko-D gloves with Hydrex significantly $(P<0.01)$ retarded the ignition time compared with those in the unwashed state. Both the glove thickness and material type appeared to be related to ignition time.

\author{
Conclusion \\ The work presented here demonstrates the acute and varying \\ flammability of a range of dental procedure gloves. It should \\ serve as a reminder to those who routinely use open flames whilst \\ gloved of the dangers of this practice.
}

In Brief

- Glove wearing blunts the temperature perception of the wearer.

- Dental gloves ignite rapidly and burn intensely.

- Reduced operator temperature perception and rapid glove ignition times leave little margin for the operator to react if the glove is exposed to a naked flame. Serious injury may result.

- Both glove material type and thickness influence the ignition time.

- Exposure to a naked flame whilst gloved should be avoided.

\section{Comment}

$A s$ dentists seek to make dentistry Abetter and safer for their patients, it sometimes happens that they make it less safe for themselves. This has been the case in one aspect of cross-infection control. The wearing of dental procedure gloves is now routine, and most dentists (and patients) feel more confident when they are used. Nevertheless, glove material is inflammable and as dentists work with naked flames the hazard of burned hands is an ever-present possibility.

The investigation carried out by Chadwick and Tulley concerns itself with this problem and scrutinises the flammability, under standardised conditions, of several makes of dental procedure glove manufactured from a variety of materials. Five types of glove were investigated both in the 'as supplied' state, and after washing with a hand disinfectant. Each was experimentally exposed to a standardised naked flame from a butane gas burner. A comparison was made of their flammability in relation to their thickness, and whether or not they had been used in conjunction with a hand antiseptic rinse.

The results were shocking, in that under the experimental conditions no glove resisted a naked flame for more than 2.5 seconds - some for less than 1.0 second. A relationship was confirmed between the thickness of the glove and the length of time before a glove ignited; thicker gloves (Biogel-D) tended to be more resistant to ignition. Use of an antiseptic glove wash also had an effect but this was variable, in some cases its use gave extra protection, extending the period of resistance by up to 0.5 seconds, in others the reverse occurred.

In their discussion the authors draw attention to the fact that surgical gloves are unlikely to satisfy the relevant safety regulations and emphasise the risks involved when, with gloved hands, dentists engage in activities which involve the use of a naked flame eg endodontics and prosthodontics. Dentists are advised that the use of a hot air burner may be a safer alternative.

\section{David J Lamb}

Senior Lecturer/Honorary Consultant, Sheffield School of Clinical Dentistry, University of Sheffield 